\title{
Propriedades ópticas lineares e não-lineares de nanopartículas de ouro superconcentradas
}

\author{
Malagón, L. A. G. \\ Escola Politécnica de Pernambuco \\ Universidade de Pernambuco \\ 50.720-001 - Recife, Brasil \\ cabm@dsc.upe.br
}

\author{
Damasceno, R. P. D. \\ Escola Politécnica de Pernambuco \\ Universidade de Pernambuco \\ 50.720-001 - Recife, Brasil
}

Resumo As propriedades lineares e não lineares (NL) ópticas de materiais compósitos contendo alta concentração de nanopartículas (NPS) de ouro foram estudados usando o modelo de Maxwell-Garnett e o modelo do gás-elétron degenerado. Foram observados altos valores do indice de refracção linear do compósito, deslocamento não-linear do pico de ressonância do plasmon e sinal de reversão das partes real e imaginária da susceptibilidade não-linear de terceira ordem. Figuras de mérito para dispositivos fotônicos foram calculadas e preenchidas tomando como base o fator de preenchimentoo e o tamanho das NPs.

\footnotetext{
Abstract Linear and nonlinear (NL) optical properties of composite materials containing high concentration of gold nanoparticles (NPs) were studied using the Maxwell-Garnett model and the degenerated electron gas model. High values of the linear refraction index of the composite, NL shift of the plasmon resonance peak and reversal sign of the real and imaginary parts of the NL third-order susceptibility were observed. Figures of merit for photonic devices were calculated and fulfilled depending of the filling factor and NPs size.
} 


\section{Introdução}

Materiais compósitos contendo nanopartículas metálicas têm recebido uma extraordinária atenção devido a seus altos valores de susceptibilidades ópticas NL $[1,2]$. Em materiais compósitos com uma topologia de Maxwell-Garnett, observa-se que a resposta óptica depende de muitas variáveis, como formato e tamanho das NPs, constante dieléctrica dos constituintes, fator de preenchimento, suscetibilidades NL de inclusão e do substrato, e o comprimento de onda e duração do pulso do laser de excitação. A influência de cada parâmetro nas propriedades ópticas lineares e NL foi estudada a partir de um ponto de vista teórico e experimental. Por exemplo, a relação entre a resposta NL e o tamanho das NPs foi recentemente estudada para NPs de prata e ouro utilizando o modelo do gás-elétron degenerado [3-5]. A influência da constante dielétrica do substrato na susceptibilidade NL de terceira ordem foi estudada e analisada usando o modelo de Maxwell-Garnett [6, 7]. Além disso, diferenças na resposta NL foram observadas sob diferentes comprimento de onda e duração do pulso do laser de excitação em compósitos contendo nanopartículas de ouro [8]. A influência do fator de preenchimento no limite de baixas concentrações foi estudada, e observou-se os cancelamentos entre a refração NL e a absorção NL com o aumento da fator de preenchimento [9, 10]. Em ambos os casos, a contribuição das propriedades ópticas NL do substrato desempenhou um papel importante na descrição sinal de reversão. No caso de compósitos contendo altas concentrações de NPs, as propriedades ópticas NL foram estudadas experimentalmente em regime de femtosegundo, picossegundo e nanossegundo $[8,11$, 12].

Vamos, aqui, analisar teoricamente a influência de altos valores do fator de preenchimento nas propriedades ópticas lineares e NL de nanopartículas de ouro utilizando o modelo do gás-elétron degenerado e o modelo de Maxwell-Garnett.

\section{Propriedades ópticas lineares}

Considere-se um compósito contendo NPs metálicas de formato esférico em um substrato com resposta óptica desprezível. As propriedades ópticas lineares podem ser estudadas usando o modelo de Maxwell-Garnett, que estabelece a relação entre a constante dielétrica do material compósito, $\varepsilon$, com as constantes dielétricas das NPs $\varepsilon_{\mathrm{NP}}$, e do substrato, $\varepsilon_{\mathrm{h}}$, da seguinte maneira:

$$
\varepsilon=\varepsilon_{\mathrm{h}}\left(1+\frac{3 \beta p}{1-\beta p}\right)
$$

em que $\beta=\left(\varepsilon_{\mathrm{NP}}-\varepsilon_{\mathrm{h}}\right) /\left(\varepsilon_{\mathrm{NP}}+2 \varepsilon_{\mathrm{h}}\right)$ e $p$ é o fator de preenchimento. O coeficiente de absorção linear, $\alpha$, e o índice de refração linear, $n$, podem ser expressos da seguinte forma:

$$
\begin{aligned}
& n=\sqrt{\frac{\varepsilon^{\prime}+\sqrt{\varepsilon^{\prime 2}+\varepsilon^{\prime \prime 2}}}{2}} \\
& \alpha=\frac{2 \pi \varepsilon^{\prime \prime}}{\lambda} \frac{\varepsilon^{n}}{}
\end{aligned}
$$

em que $\lambda$ é o comprimento de onda, e $\varepsilon$ ' e $\varepsilon$ "' são as partes real e imaginária de $\varepsilon$, respectivamente. A dependência entre a constante dielétrica das NPs e a frequência e tamanho foi calculada anteriormente para NPs de ouro usando o modelo do Ponto Crítico incluindo correções no parâmetro de amortecimento [3]. Assim, a constante dielétrica das NPs pode ser escrita como:

$\varepsilon_{\mathrm{NP}}(\omega, R)=\varepsilon_{\mathrm{C} . \mathrm{P}}(\omega)+i \frac{A \omega_{\mathrm{p}}^{2} v_{\mathrm{F}}}{\omega^{3} R}$

em que $\varepsilon_{C . P}(\omega)$ é a constante dielétrica do ouro em função da frequência calculada utilizando o modelo do Ponto Crítico, $A$ é o parâmetro de dispersão, $\omega_{\mathrm{p}}$ é a frequência de plasma, $\nu_{\mathrm{F}}$ é a velocidade de Fermi, $\omega=2 \pi / \lambda$ é a frequência, e R é o raio das NPs.

No limite de valores baixos do fator de preenchimento, uma expressão analítica para o coeficiente de absorção linear pode ser calculada a partir de (3) utilizando (1) e (2), como segue:

$\alpha(\lambda, R)=\frac{18 \pi p}{\lambda} \varepsilon_{\mathrm{h}}^{3 / 2} \frac{\varepsilon_{\mathrm{NP}}^{\prime \prime}(\lambda, R)}{\left(\varepsilon_{\mathrm{NP}}^{\prime}(\lambda, R)+2 \varepsilon_{\mathrm{h}}\right)^{2}+\varepsilon_{\mathrm{NP}}^{\prime \prime 2}(\lambda, R)}$.

A equação (5) mostra uma dependência linear entre o coeficiente de absorção e $p$, e um valor máximo, conhecido como ressonância de plasma, quando $\left(\varepsilon_{\mathrm{NP}}{ }_{\mathrm{N}}(\lambda, \mathrm{R})+2 \varepsilon_{\mathrm{h}}\right)^{2}+$ $\varepsilon^{\prime \prime 2}{ }_{\mathrm{NP}}(\lambda, \mathrm{R}) \approx 0$. Além disso, o índice de refração linear calculado a partir da equação (2) pode ser aproximada para $n$ $\approx \sqrt{ } \varepsilon$ ' porque $\varepsilon " \approx 0$ no espectro visível. Como a parte real da constante dieléctrica é praticamente constante, o índice de refração linear também o é.

No entanto, para valores elevados de $p$, é importante levar em consideração a contribuição das polarizações da vizinhança das NPs na resposta óptica. Tomando essa consideração, as propriedades ópticas lineares de um compósito Maxwell-Garnett com diferentes tamanhos e fatores de preenchimento para NPs de ouro em um substrato vítreo $\left(\varepsilon_{\mathrm{h}}=2,25\right)$ foram calculadas usando as Equações (1) - (3), e os resultados são mostrados nas Figuras 1, 2, 3 e 4.

Os espectros do coeficiente de absorção linear espectros são mostrados na Fig. 1, para diferentes valores de $p$. 


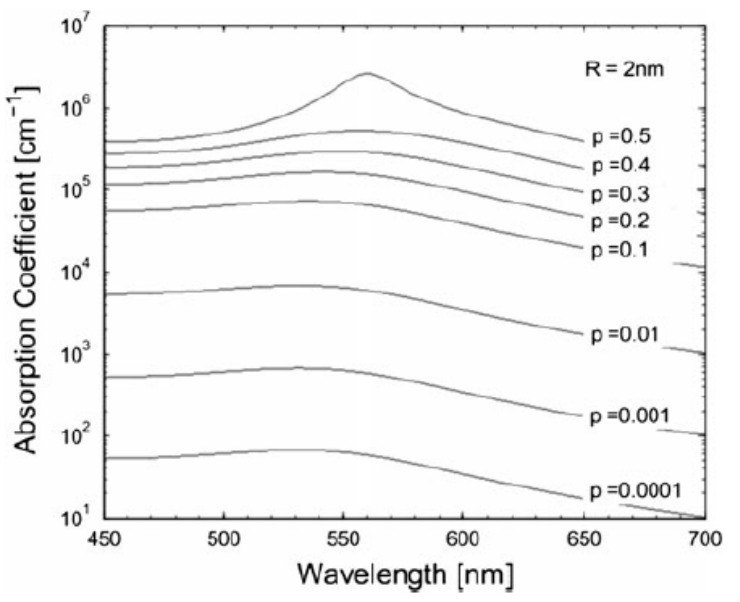

Fig 1. Dependência espectral do coeficiente de absorção para diferentes valores do fator de preenchimento.

A partir desses espectros, observa-se um deslocamento vermelho no pico de ressonância do plasmon (PRP) quando o fator de preenchimento é aumentado. A dependência entre o PRP e o tamanho das NPs e $p$ é apresentada na Fig. 2.

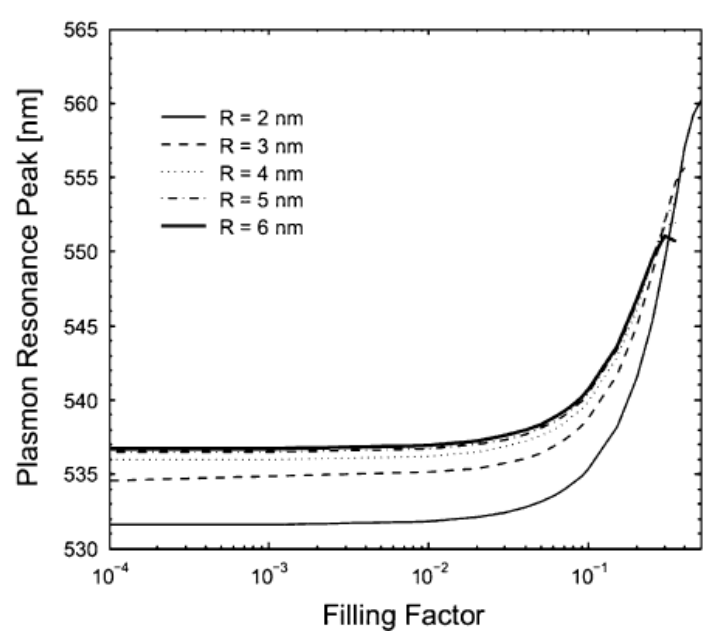

Fig 2. Dependência do pico de ressonância do plasmon e o fator de preenchimento para vários tamanhos de NPs.

O PRP é deslocado em vermelho quando o tamanho das NPs é aumentado e tem uma forte variação NL para $p$ $>0,01$. Para $\mathrm{p}<0,01$, observa-se que o PRP é praticamente constante para um compósito com NPs de tamanho constante. Por outro lado, a dependência entre o índice de refração linear e o fator de preenchimento e tamanho das NPs é mostrada nas Figs. 3 e 4.

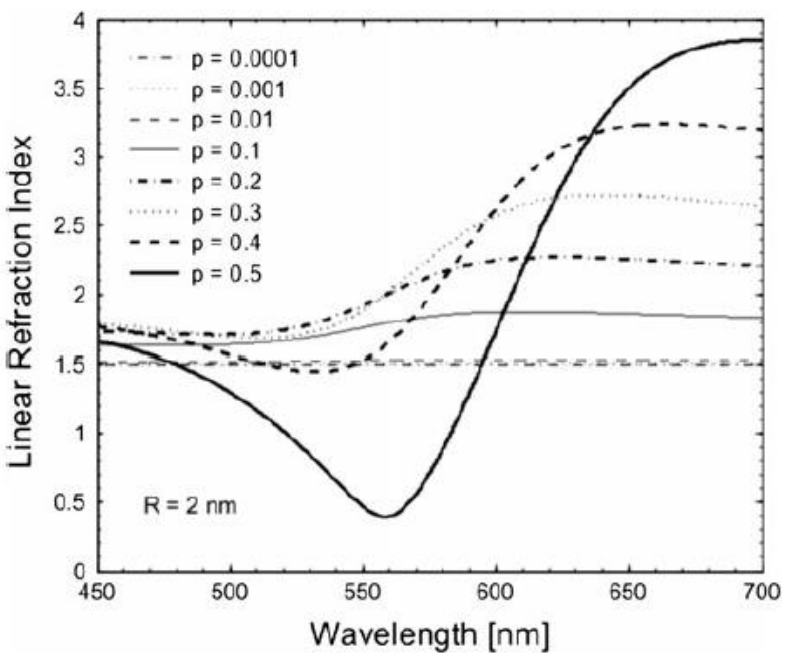

Fig 3. Dependência espectral do índice de refração linear para diferentes valores do fator de preenchimento.

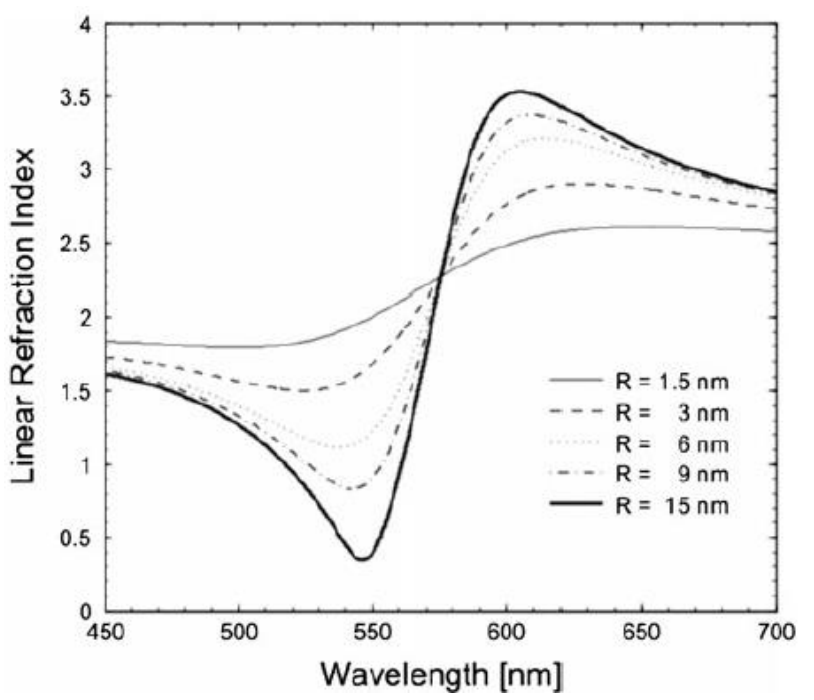

Fig 4. Dependência espectral do índice de refração linear para vários tamanhos de NPs.

Para baixos valores de $p$, o índice de refração linear é praticamente constante no espectro visível tal como apresentado na Fig. 3. Para valores elevados do fator de preenchimento, foram previstos altos e baixos valores no índice de refração linear em torno da ressonância de plasma e próximo à região do infravermelho, respectivamente. Na Fig. 4 , a dispersão do índice de refração linear foi calculada considerando constante o valor de $p(p=0,3)$ e aumentando o tamanho das NPs. Da figura vê-se que o índice de refração linear tem valores menores que $1 \mathrm{em}$ torno do PRP, permitindo altos valores no coeficiente de absorção de acordo com a Equação (3). Além disso, para comprimentos de onda em torno de $600 \mathrm{~nm}$, o índice de refração linear aumenta com o tamanho das NPs, indicando elevada resposta não linear de acordo com a regra da Miller [13]. 
A partir da Fig. 3, é importante observar que, se o fator de preenchimento é aumentado ainda mais, pode-se obter valores negativos de $n$ conforme relatado para nanoestruturas similares [14-16]. Do ponto de vista experimental, medições das propriedades ópticas lineares de compósitos que contenham NPs de ouro são relatadas na literatura, e o modelo de Maxwell-Garnett aqui apresentado descreve bem os resultados publicados [17-19].

\section{Propriedades ópticas não-lineares}

Para um compósito com uma topologia de MaxwellGarnett e resposta óptica do substrato desprezível, as propriedades ópticas NL podem ser calculadas utilizando o modelo de Maxwell-Garnett e o modelo do gás-elétron degenerado. Para baixos fatores de preenchimento, e considerando que o compósito está na presença de um campo elétrico externo $\mathrm{E}_{0}$, o campo elétrico dentro da NP é $\mathrm{E}_{\text {in }}=$ $f \mathrm{E}_{\mathrm{o}}$, em que o termo $f=3 \varepsilon_{\mathrm{h}} /\left(\varepsilon_{\mathrm{NP}}+2 \varepsilon_{\mathrm{h}}\right)$ é o fator de campo local. Sob esta condição, a suscetibilidade NL de terceira ordem do compósito $\chi_{\text {eff }}^{(3)}$, pode ser escrita como $\chi_{\text {eff }}^{(3)}=p f^{2}|f|^{2} \chi_{\mathrm{NP}}^{(3)}$, em que $\chi_{\mathrm{NP}}^{(3)}$ é a suscetibilidade NL de terceira ordem das NPs. Utilizando esta abordagem, as propriedades ópticas NL foram calculadas para os compósitos contendo NPs de prata e de ouro $[3,4]$. No entanto, para valores elevados de $p$, o campo eléctrico em torno da NP é afetado pelas contribuições das outras NPs. Dessa forma, o fator de campo local é corrigido e a susceptibilidade NL pode ser escrita como $\chi_{\mathrm{eff}}^{(3)}=p \eta^{2}|\eta|^{2} \chi_{\mathrm{NP}}^{(3)}$, em que $\eta=\left(\varepsilon+2 \varepsilon_{\mathrm{h}}\right) /\left(\varepsilon_{\mathrm{NP}}+2 \varepsilon_{\mathrm{h}}\right)[20]$. A partir desta expressão, a dependência NL de $\chi_{\text {eff }}^{(3)} \operatorname{com} p$ é dada pelo fator de campo local corrigido, $\eta$.

A suscetibilidade NL de terceira ordem das NPs pode ser calculada usando o modelo do gás-elétron degenerado, que descreve $\chi_{\mathrm{NP}}^{(3)}$ considerando que um elétron está em uma fonte de potencial esférica infinitamente profunda de raio R. No limite de $l \gg(R / \lambda) \gg\left(v_{\mathrm{F}} / 2 \pi c\right), \chi_{\mathrm{NP}}^{(3)}$ pode ser escrita como [5]:

$$
\begin{aligned}
\chi_{\mathrm{NP}}^{(3)}= & \frac{2}{15}\left(\frac{e^{2} n}{m \omega^{2}}\right)\left(\frac{e R}{\hbar \omega}\right)^{2} \frac{\Gamma_{2}}{\Gamma_{1}} \\
& \times\left\{F_{3}-i\left[\frac{2 \Gamma_{2}}{\omega} F_{3}+\left(\frac{\omega}{2 \Gamma_{2}}\right)^{2}\left(\frac{v_{\mathrm{F}}}{R \omega}\right)^{5} g_{3}\right]\right\}
\end{aligned}
$$

em que $m$ é a massa do elétron, $e$ representa sua carga, $\mathrm{n}$ é a densidade de elétrons, $\omega=2 \pi \mathrm{c} / \lambda$ é a frequência, $c$ é a velocidade da luz, $\hbar$ é a constante de Planck reduzida, $v_{\mathrm{F}}$ é a velocidade de Fermi, $F_{3}$ varia de 0,30 a 0,33 para partículas entre 2 e $15 \mathrm{~nm}, g_{3}=0,64$, e $\Gamma_{1}$ e $\Gamma_{2}$ representam as taxas de relaxamento para a população e coerência, respectivamente. Os valores de $\Gamma_{1}$ e $\Gamma_{2}$ foram determinados anteriormente para compósitos contendo NPs de ouro [3].
Os cálculos das propriedades ópticas NL de um compósito contendo NPs de ouro com raios de $3 \mathrm{~nm}$ incorporadas em um substrato com um índice de refração linear de 1,5 são dados nas Fig. 5 e 6.

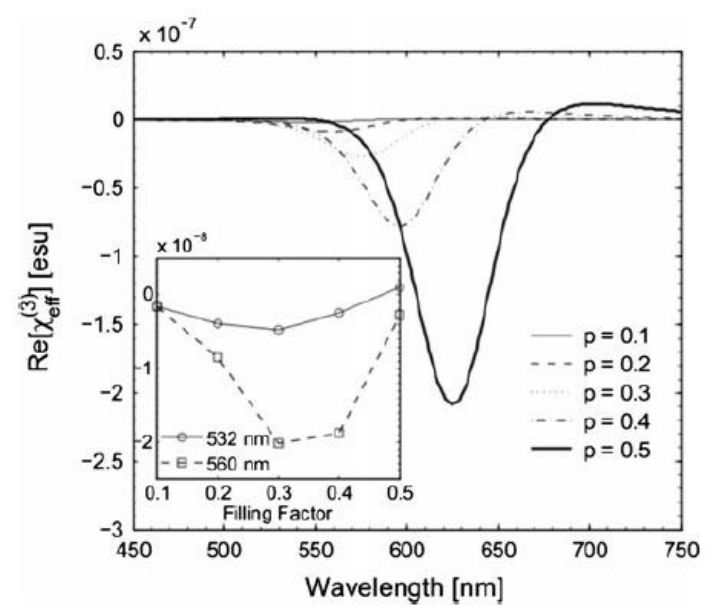

Fig 5. Dependência espectral da parte real da suscetibilidade NL de terceira ordem para diferentes valores do fator de preenchimento.

Os resultados mostram mudanças no sinal da parte real e imaginária da susceptibilidade NL. Por exemplo, em 532 $\mathrm{nm}$ (560 nm), há uma mudança na parte real (imaginária) de negativo (positivo) para positivo (negativo), enquanto que a parte imaginária (real) tem um comportamento NL sem reversão do sinal. Mudanças de quase três ordens de magnitude para as partes imaginária e real da suscetibilidade NL em 650 e $625 \mathrm{~nm}$ de $1,8 \times 10^{-10}$ e $3.0 \times 10^{-10}$ esu para $p=0,1-1,31 \times 10^{-07} \mathrm{e}-2.1 \times 10^{-07}$ esu para $p=0,5$ foram previstos, respectivamente.

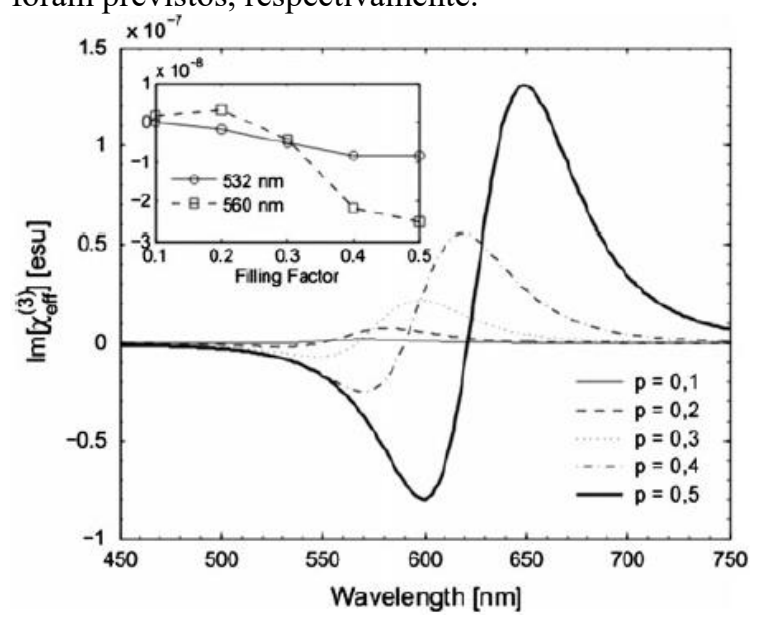

Fig 6. Dependência espectral da parte imaginária da suscetibilidade NL de terceira ordem para diferentes valores do fator de preenchimento. 
A influência do tamanho das NPs na resposta NL foi calculada para $p=0,3$ e os resultados são apresentados nas Figs. 7 e 8.

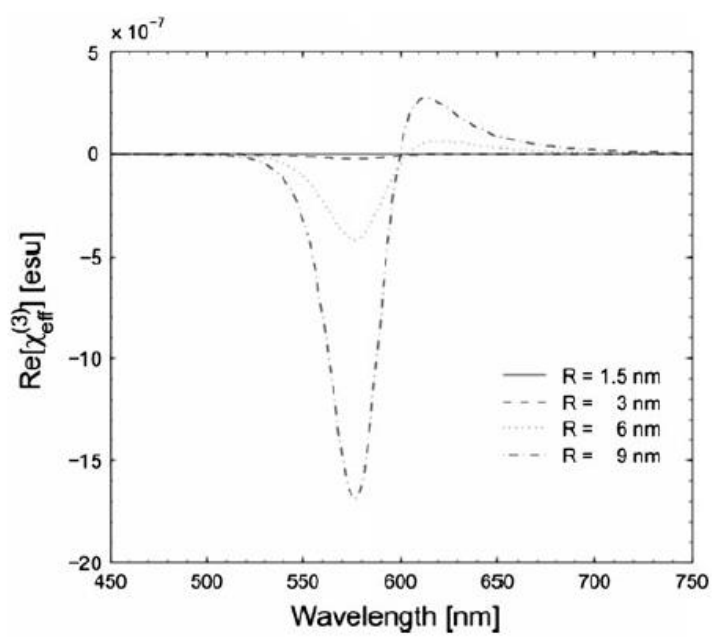

Fig 7. Dependência espectral da parte real da suscetibilidade NL de terceira ordem para vários tamanhos de NPs.

Das figuras, observa-se que a posição da resposta máxima não é alterada, e que a resposta NL é aumentada de forma não linear, quando o tamanho das NPs cresce. A dependência de $x_{\text {crr }}^{(3)} \operatorname{com} R$ é dada através das Eqs. 4 e 5 para $\varepsilon_{\mathrm{NP}}(\omega, \mathrm{R})$ e $\chi_{\mathrm{NP}}^{(3)}$, respectivamente.

Para compreender a influência do fator de preenchimento na resposta NL, é importante observar que o campo local corrigido pode ser escrito como $\eta=f /(1-\beta p)$, em que o termo $1 /(1-\beta p)$ é o fator de correção de $f$. Portanto, as diferenças entre a

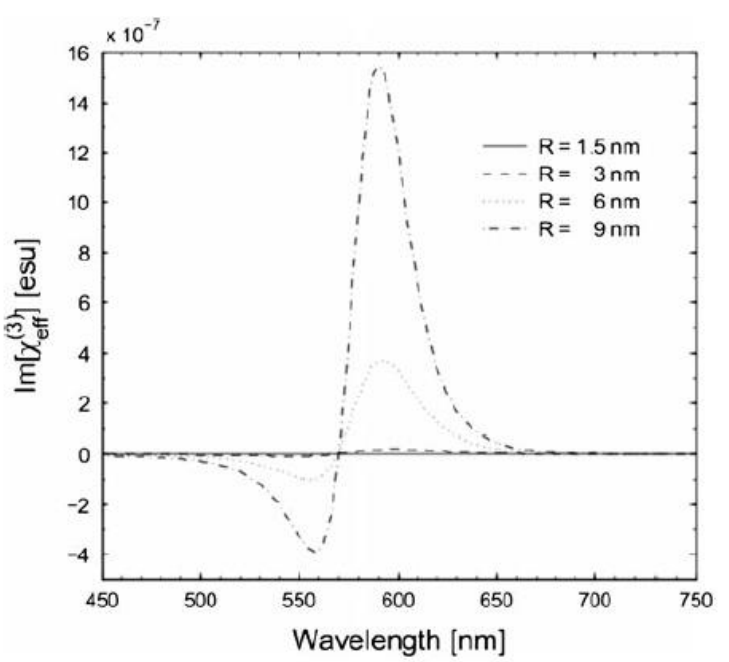

Fig 8. Dependência espectral da parte imaginária da suscetibilidade NL de terceira ordem para vários tamanhos de NPs. susceptibilidade NL em altos e baixos valores do fator de preenchimento são dadas pelo quociente $\chi_{\text {eff,high }}^{(3)} / \chi_{\text {eff,low }}^{(3)}=(1-\beta p)^{2}|(1-\beta p)|^{2}$. Em pequenos valores de $p$, o termo $1-\beta p$ é praticamente real e o sinal da susceptibilidade NL do compósito é dado pela susceptibilidade NL das NPs. No entanto, em valores elevados de $p$, a parte imaginária de $1-\beta p$ não é desprezível e será responsável pela dependência entre as partes real e imaginária de $\chi_{\text {eff }}^{(3)}$ e $p$.

Do ponto de vista experimental, a Fig. 9 mostra os valores medidos de $\left|\chi_{\text {eff }}^{(3)}\right|$ obtidos no regime femtosegundo em $532 \mathrm{~nm}$ para compósitos com altos valores do fator de preenchimento de NPs de ouro em $\mathrm{SiO}_{2}$ [8].

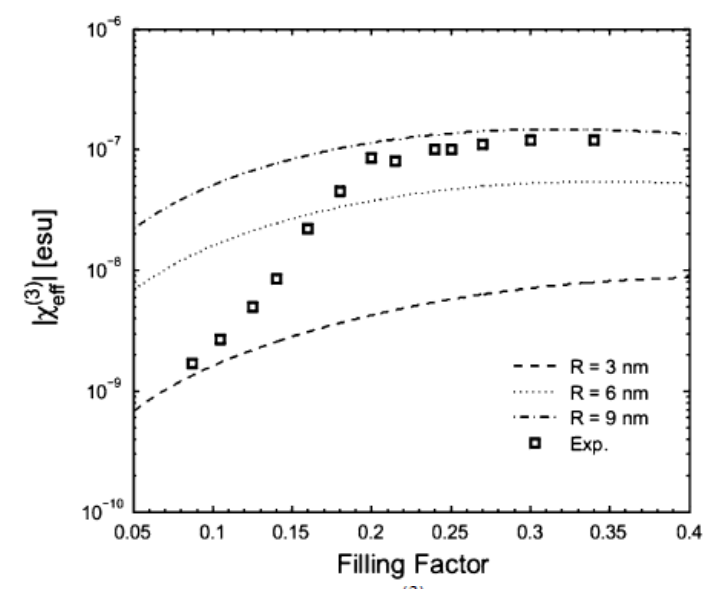

Fig 9. Dependência entre $\left|\chi_{\text {eff }}^{(3)}\right|$ e o fator de preenchimento para diferentes tamanhos de NPs. Valores experimentais de [7].

Assim, com o experimento, as amostras com elevados valores de $p$ foram obtidos por co-pulverização de Au e $\mathrm{SiO}_{2}$ em um sistema denominado multitarget magnetron sputtering. Os filmes de $\mathrm{Au}: \mathrm{SiO}_{2}$ foram depositados sobre um substrato de quartzo fundido em $150^{\circ} \mathrm{C}$ numa atmosfera de Ar. Imediatamente após a deposição, as amostras com diferentes concentrações de $\mathrm{Au}$ foram recozidas a $850^{\circ} \mathrm{C}$ durante $1 \mathrm{~min}$, num forno de recozimento rápido na presença de gás Ar a 1 atm, e em seguida resfriadas num fluxo de atmosfera de Ar. O tamanho médio das NPs de ouro foi determinado utilizando os dados de difração de raios X e a equação de Scherrer. Foram esperadas NPs pequenas com tamanho inferior a $3 \mathrm{~nm}$ para $\mathrm{p}<0,25$, e para fatores de preenchimento maiores que 0,25 , o tamanho das NPs de ouro ficou no intervalo de 3 a $80 \mathrm{~nm}$. Aumentando o fator de preenchimento, o PRP deslocou para o vermelho devido ao aumento do tamanho das NPs de ouro [21]. Imagens de TEM dessas amostras não foram publicadas; no entanto, imagens de TEM de filmes de $\mathrm{Au}: \mathrm{Al}_{2} \mathrm{O}_{3}$ fabricados usando a mesma técnica revela que o tamanho das NPs é semelhante para os filmes de $\mathrm{Au}: \mathrm{SiO}_{2}$ na mesma faixa de 
concentrações [22]. Para comparação, a Fig. 9 também mostra os valores calculados de $\left|\chi_{\text {eff }}^{(3)}\right|$ para diferentes tamanhos de NPs. A partir da figura, observa-se que os valores medidos de $\left|\chi_{\text {eff }}^{(3)}\right|$ estão no intervalo de $\left|\chi_{\text {eff }}^{(3)}\right|$ calculado.

Como o modelo pode descrever a susceptibilidade NL de terceira ordem de NPs de ouro altamente concentradas, aplicações em fotônica podem ser exploradas através das figuras de mérito. Para dispositivos de comutação óptica, $T=2 \alpha_{2} \lambda / n_{2}<1$ deve ser satisfeito, em que $\alpha_{2}$ e $n_{2}$ são os coeficientes de absorção NL e de refração NL, respectivamente. As relações entre os coeficientes NL e as partes real e imaginária da susceptibilidade NL são dadas por $n_{2}=\frac{3}{4 \varepsilon_{0} n^{2} c^{2}} \operatorname{Re}\left[\chi_{\text {eff }}^{(3)}\right]$ e $\alpha_{2}=\frac{3 \omega}{2 \varepsilon_{0} n^{2} c} \operatorname{Im}\left[\chi_{\text {eff }}^{(3)}\right]$, em que $\varepsilon_{0}$ é a permissividade do vácuo. Portanto, $T=8 \pi \frac{\operatorname{Im}\left[\chi_{\text {eff }}^{(3)}\right]}{\operatorname{Re}\left[\chi_{\text {eff }}^{(3)}\right]}<1$ [23]. Para satisfazer essa relação, o valor de $\operatorname{Im}\left[\chi_{\text {eff }}^{(3)}\right]$ deve estar próximo ao zero, o que pode ser observado na Fig. 6 em 532 nm quando o sinal de reversão de $\operatorname{Im}\left[\chi_{\text {eff }}^{(3)}\right]$ é obtido para fatores de preenchimento entre 0,2 e 0,3 . Para outros comprimentos de onda, é possível ajustar a curva de dispersão alterando o valor de $p$ para obter $\operatorname{Im}\left[\chi_{\text {eff }}^{(3)}\right] \approx 0$.

Além disso, para um dispositivo de fotônica, espera-se ter um material com elevado valor de $\left|\chi_{\text {eff }}^{(3)}\right|$ e de baixo valor de $\alpha$. Assim, a figura de mérito $\left|\chi_{\text {eff }}^{(3)}\right| / \alpha$ foi calculada em 532 nm como uma função do fator de preenchimento para os diferentes tamanho de NPs, e os resultados são mostrados na Fig. 10.

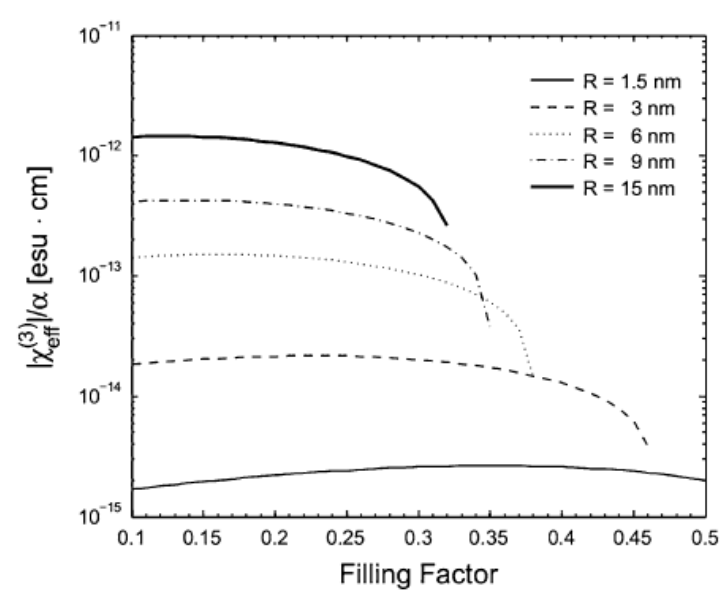

Fig 10. Dependência entre $\left|\chi_{\text {eff }}^{(3)}\right| / \alpha$ e o fator de preenchimento para diferentes tamanhos de NPs.

Resultados semelhantes foram obtidos para compósitos de $\mathrm{Au}: \mathrm{SiO}_{2}$ excitados com laser de picosegundo [21]. A partir da figura, observa-se que a figura de mérito pode ser calculada até um valor limite do fator de preenchimento dependendo do tamanho das NPs. Este limite é definido pelo coeficiente de absorção linear e pelo índice de refração linear que foram calculados usando o modelo de Maxwell-Garnett.

\section{Conclusão}

Os cálculos das propriedades ópticas lineares usando o modelo de Maxwell-Garnett revelam o limite de aplicabilidade do modelo com base na dimensão das NPs. Para tamanhos pequenos, as propriedades ópticas lineares podem ser calculadas para fatores de preenchimento maiores do que para as grandes NPS. A susceptibilidade de terceira ordem NL calculada utilizando o modelo de Maxwell-Garnett e o modelo do gás-elétron degenerado está de acordo com os valores experimentais relatados na literatura, e prevê a reversão de sinal da resposta NL dependendo do fator de preenchimento. Para compósitos com alta concentração de NPs de ouro, os requisitos dados pelas figuras de mérito para dispositivos ópticos podem ser atendidos dependendo do fator de preenchimento e do tamanho das NPs.

Agradecimentos $\mathrm{O}$ apoio financeiro proveniente das agências brasileiras Conselho Nacional de Desenvolvimento Científico e Tecnológico (CNPq), Fundação de Amparo à Ciência e Tecnologia do Estado de Pernambuco (FACEPE) e Escola Politécnica de Pernambuco (POLIUPE) é reconhecido. Este trabalho foi realizado sob o Projeto PRONEX.

\section{Referências}

[1] F. Hache, D. Ricard, C. Flytzanis, J. Opt. Soc. Am. B 3, 1647-1655 (1986)

[2] F. Hache, D. Ricard, C. Flytzanis, U. Kreibig, Appl. Phys. A 47, 347-357 (1988)

[3] J.B. Monteiro-Filho, L.A. Gómez-Malagón, J. Opt. Soc. Am. B 29, 1793-1798 (2012)

[4] V.P. Drachev, A.K. Buin, H. Nakotte, V.M. Shalaev, Nano Lett. 4, 1535-1539 (2004)

[5] S.G. Rautian, JETP 85, 451 (1997)

[6] L.A. Gómez, C.B. de Araújo, A.M. Brito Silva, A. Galembeck, Appl. Phys. B 92, 61-66 (2008)

[7] R.A. Ganeev, M. Baba, A.I. Ryasnyansky, M. Suzuki, H. Kuroda, Opt. Commun. 240, 437-448 (2004) 
[8] H.B. Liao, R.F. Xiao, J.S. Fu, H. Wang, K.S. Wong, G.K.L. Wong, Opt. Lett. 23, 388-390 (1998)

[9] E.L. Falcão-Filho, C.B. de Araújo, A. Galembeck, M.M. Oliveira, A.J.G. Zarbin, J. Opt. Soc. Am. B 22, 2444-2449 (2005)

[10] D.D. Smith, G. Fischer, R.W. Boyd, D.A. Gregory, J. Opt. Soc. Am. B 14, 1625-1631 (1997)

[11] H.B. Liao, W. Wen, G.K.L. Wong, Appl. Phys. A $80,861-864$ (2005)

[12] N. Pinc, on, B. Palpant, D. Prot, E. Charron, S. Debrus, Eur. Phys. J. D 19, 395-402 (2002)

[13] R. Boyd, Nonlinear Optics (Academic Press, San Diego, 2008)

[14] S. Riikonen, I. Romero, F.J. García de Abajo, Phys. Rev. B 71, 235104 (2005)

[15] J.A. Fan, C. Wu, K. Bao, J. Bao, R. Bardhan, N.J. Halas, V.N. Manoharan, P. Nordlander, G. Shvets, F. Capasso, Science 328, 1135-1138 (2010)

[16] R. Paniagua-Domínguez, F. López-Tejeira, R. Marque's, J.A. Sa'nchez-Gil, New J. Phys. 13, 123017 (2011)

[17] Y. Takeda, O.A. Plaksin, N. Kishimoto, Opt. Express 15, 6010-6018 (2007)

[18] S. Link, M.A. El-Sayed, J. Phys. Chem. B103, 8410-8426 (1999)

[19] G. Ma, W. Sun, S. Tang, H. Zhang, Z. Shen, Opt. Lett. 24, 1043-1045 (2002)

[20] J.E. Sipe, R.W. Boyd, Phys. Rev. A 46, 16141629 (1992)

[21] H.B. Liao, R.F. Xiao, J.S. Fu, P. Yu, G.K.L. Wong, P. Shen, Appl. Phys. Lett. 70, 1-3 (1997)

[22] H.B. Liao, R.F. Xiao, J.S. Fu, G.K.L. Wong, Appl. Phys. B 65, 673-676 (1997)

[23] V. Mizrahi, K.W. DeLong, G.I. Stegeman, M.A. Saifi, M.J. Andrejco, Opt. Lett. 14, 1140-1142 (1989) 